\title{
GLS hyperactivity causes glutamate excess, infantile cataract and profound developmental delay
}

DOI:

10.1093/hmg/ddy330

\section{Document Version}

Accepted author manuscript

Link to publication record in Manchester Research Explorer

\section{Citation for published version (APA):}

Taylor, R. L., \& Black, G. (2019). GLS hyperactivity causes glutamate excess, infantile cataract and profound developmental delay. Human Molecular Genetics, 28(1), 96-104. https://doi.org/10.1093/hmg/ddy330

\section{Published in:}

Human Molecular Genetics

\section{Citing this paper}

Please note that where the full-text provided on Manchester Research Explorer is the Author Accepted Manuscript or Proof version this may differ from the final Published version. If citing, it is advised that you check and use the publisher's definitive version.

\section{General rights}

Copyright and moral rights for the publications made accessible in the Research Explorer are retained by the authors and/or other copyright owners and it is a condition of accessing publications that users recognise and abide by the legal requirements associated with these rights.

\section{Takedown policy}

If you believe that this document breaches copyright please refer to the University of Manchester's Takedown Procedures [http://man.ac.uk/04Y6Bo] or contact uml.scholarlycommunications@manchester.ac.uk providing relevant details, so we can investigate your claim.

\section{OPEN ACCESS}


4 Lynne Rumping ${ }^{1,2,3} \mathrm{~b}$, Federico Tessadori ${ }^{1,2,4} \mathrm{~b}$, Petra JW Pouwels ${ }^{5}$, Esmee Vringer $^{1}$, Jannie P

5 Wijnen $^{6}$, Alex A Bhogal ${ }^{6}$, Sanne MC Savelberg ${ }^{1}$, Karen J Duran ${ }^{1}$, Mark JG Bakkers ${ }^{7}$, Rúben

6 JJ Ramos ${ }^{1,2}$, Peter AW Schellekens ${ }^{8}$, Hester Y Kroes ${ }^{1}$, Dennis WJ Klomp ${ }^{6}$, Graeme CM

$7 \quad$ Black $^{9,10}$, Rachel L Taylor ${ }^{9,10}$, Jeroen PW Bakkers ${ }^{4,11}$, Hubertus CMT Prinsen ${ }^{1}$, Marjo S van

8 der Knaap ${ }^{5}$, Tobias B Dansen ${ }^{2,13}$, Holger Rehmann ${ }^{2,13}$, Fried JT Zwartkruis ${ }^{2,13}$, Roderick HJ

9 Houwen $^{3}$, Gijs van Haaften ${ }^{1,2}$, Nanda M Verhoeven-Duif ${ }^{1,2}$, Judith JM Jans ${ }^{1,2 * b}$, Peter M van

10 Hasselt $^{3 * \mathrm{p}}$

11 b Lynne Rumping and Federico Tessadori contributed equally to this work.

12 b Judith JM Jans and Peter M van Hasselt contributed equally to this work.

\section{Affiliations:}

14

$153584 \mathrm{CX}$, the Netherlands

$16{ }^{2}$ Center for Molecular Medicine, University Medical Center Utrecht, Utrecht University,

17 Utrecht $3584 \mathrm{CX}$, the Netherlands

$18{ }^{3}$ Department of Pediatrics, University Medical Center Utrecht, Utrecht University, Utrecht

$193584 \mathrm{CX}$, the Netherlands

20

${ }^{4}$ Hubrecht Institute-KNAW, University Medical Center Utrecht, Utrecht University, Utrecht

$213584 \mathrm{CT}$, the Netherlands 
${ }^{5}$ Department of Radiology and Nuclear Medicine, VU University Medical Center, Amsterdam $1081 \mathrm{HV}$, the Netherlands

${ }^{6}$ Department of Radiology, University Medical Center Utrecht, Utrecht University, Utrecht $3584 \mathrm{CX}$, the Netherlands

${ }^{7}$ Department of Microbiology and Immunobiology, Harvard Medical School, Boston MA 02115, USA

${ }^{8}$ Department of Ophthalmology, University Medical Center Utrecht, Utrecht University, Utrecht 3584 CX, the Netherlands

${ }^{9}$ Division of Evolution and Genomic Sciences, The University of Manchester, Manchester M139WL, UK

${ }^{10}$ Manchester Centre for Genomic Medicine, St Mary's Hospital, Manchester M139WL, UK

${ }^{11}$ Department of Medical Physiology, University Medical Center Utrecht, Utrecht University, Utrecht $3584 \mathrm{CX}$, the Netherlands

${ }^{12}$ Department of Child Neurology, VU University Medical Center, Amsterdam $1081 \mathrm{HV}$, the Netherlands

${ }^{13}$ Molecular Cancer Research, University Medical Center Utrecht, Utrecht University, Utrecht 3584 CX, the Netherlands

\section{Corresponding author*:}

Peter M van Hasselt, Lundlaan 3584 EA Utrecht, the Netherlands, e-mail address: p.vanhasselt@umcutrecht.nl, tel: +31 8875550 56, fax: +31 887555349 


\section{Abstract}

45 Loss-of-function mutations in glutaminase (GLS), the enzyme converting glutamine into glutamate, and the counteracting enzyme glutamine synthetase (GS) cause disturbed glutamate homeostasis and severe neonatal encephalopathy. We report a de novo Ser482Cys gain-offunction variant in GLS encoding glutaminase associated with profound developmental delay and infantile cataract. Functional analysis demonstrated that this variant causes hyperactivity and compensatory downregulation of GLS expression combined with upregulation of the counteracting enzyme GS, supporting pathogenicity. Ser482Cys-GLS likely improves the electrostatic environment of the GLS catalytic site, thereby intrinsically inducing hyperactivity. Alignment of +/-12.000 GLS protein sequences from $>1000$ genera, revealed extreme conservation of Ser482, to the same degree as catalytic residues. Together with the hyperactivity, this indicates that Ser482 is evolutionarily preserved to achieve optimal -but submaximal- GLS activity. In line with GLS hyperactivity, increased glutamate and decreased glutamine concentrations were measured in urine and fibroblasts. In the brain (both grey and white matter), glutamate was also extremely high and glutamine almost undetectable, using ultra-high field magnetic resonance spectroscopic imaging. Considering the neurotoxicity of glutamate when present in excess, the strikingly high glutamate concentrations measured in the brain provide an explanation for the developmental delay. Cataract, a known consequence of oxidative stress, was evoked in zebrafish expressing the hypermorphic Ser482Cys-GLS and could be alleviated by inhibition of GLS. The capacity to detoxify reactive oxygen species was reduced upon Ser482Cys-GLS expression, providing an explanation for cataract formation. In conclusion, we describe an inborn error of glutamate metabolism caused by a GLS hyperactivity variant, illustrating the importance of balanced GLS activity.

\section{Introduction}


The amino acid glutamate is best known for its role as excitatory neurotransmitter, but also serves as a substrate for other key metabolites, including the anti-oxidant glutathione(1-3). Glutamate homeostasis is mainly warranted by two enzymes: glutamine synthetase (GS; EC 6.3.1.2) and glutaminase (GLS; EC 3.5.1.2). GS converts glutamate into glutamine and is ubiquitously expressed(4). GLS catalyzes the deamination of glutamine into glutamate and ammonia and exists in two isoforms: GLS -present in two splice variants KGA and GACmainly expressed in kidney and brain; and GLS2, ubiquitously expressed with the highest expression in the liver $(5,6)$.

Inborn errors of metabolism are usually due to severe loss of function of the involved enzymes, hence recessive inheritance. In line, a disturbed equilibrium of glutamate and glutamine was described in patients with GS deficiency, clinically resulting in glutamine deficiency, neonatal epilepsy and early death(7). Recently, GLS loss of function has been described to cause lethal epileptic encephalopathy and glutamine excess in two families(8). The description of patients with spastic ataxia and optic atrophy harbouring bi-allelic hypomorphic variants in GLS, suggests a phenotypic spectrum -presumably depending on the degree of residual activity- that is yet to be uncovered(9). Theoretically, glutamate homeostasis can also be disturbed by hyperactivity of either enzyme. This option is commonly disregarded as there are only few examples of genetic variants that induce enzyme hyperactivity, GDH and IDH2 gain of functions $(10,11)$. These examples indicate that a heterozygous variant is sufficient to induce overall enzyme hyperactivity.

In this study, we characterize a de novo hypermorphic heterozygous GLS variant found in a patient with infantile onset cataract, skin abnormalities, profound developmental delay and intracerebral glutamate excess. This new inborn error of metabolism illustrates the importance of regulated GLS activity for lens transparency and brain function. 


\section{Results}

\section{Clinical description}

In a female patient, bilateral cataract was diagnosed at the age of 3 months after the parents noticed decreased eye contact and loss of the red light reflex of the pupils on photos (Figure. 1A, S1). The proband is the first child of healthy non-consanguineous parents of Dutch descent (Figure. S1A) as indicated by family history and SNP-array. Gestation and delivery were uneventful. After lens extraction and replacement, eye contact unexpectedly remained absent. By the age of 8 months, delayed development was noted, along with a relative decrease of the head circumference from 0 SD to -2 SD. She developed recurrent dermatological abnormalities on her extremities, cheeks and ears without pruritus, characterized as erythematic subcutaneous nodules of approximately $1 \mathrm{~cm}$ (Figure. 1B). Histopathological analysis of these lesions showed deep perivascular and periglandular lymphohistiocytic infiltrates and pronounced leukocytoclasia at the surface of the dermis and focal vacuolar alterations, hyperkeratosis and parakeratosis of the epidermis. A dermatological diagnosis remained inconclusive. Over time, the girl lost the ability to make meaningful sounds and the ability to sit. She developed profound axial hypotonia leading to kyphoscoliosis. Upon arousal she exhibited uncontrolled motoric agitation and self-injurious behavior. Development remained slow paced. At the most recent follow up at the age of 11 years, she was able to use gestures for communication, to understand verbal single component instructions and to steer her own wheelchair.

\section{Identification of the Ser482Cys-GLS de novo variant}

Extensive diagnostic workup unexpectedly revealed extremely low glutamine levels and high glutamate levels in both cortex and white matter as detected consistently with quantitative brain proton MRS and MRSI at 1.5Tesla (Figure. 1C, S1B) and recently also shown at 7Tesla (Figure 1D, S1C). Interestingly, CSF and plasma levels were unaffected (Table S1). Brain MRI at age 16 months showed delayed myelination (Figure 1E). Analyses of stored urine samples similarly 
118 showed low concentrations of glutamine and high concentrations of glutamate (Figure. 1F,

119 Table S1). The diagnosis remained enigmatic until trio-based whole exome sequencing (WES)

120 revealed a heterozygous de novo GLS missense variant (NC_000002.11:g.191795182C>G).

121 Analysis of WES data using recessive filters yielded no rare homozygous damaging variants.

122 The analysis for compound heterozygosity (including correctness of segregation in parents)

123 yielded two genes hit by rare and possibly damaging variants, but based on gene function,

124 absent links with human disease and the high prevalence within the healthy population, these

125 variants were considered as unlikely to contribute to the phenotypes of the patient

126 (supplementary results). The conservative mutation in GLS from serine to cysteine at position

127482 NP_055720.3:p.(Ser482Cys) was confirmed by Sanger sequencing (Figure. 1G,

128 supplementary results). Glutaminase mediates the conversion of glutamine into glutamate,

129 therefore this genetic change could only be explained if the encoded protein would be 130 hyperactive.

Ser482Cys-GLS leads to GLS hyperactivity

133 The effect of the Ser482Cys-GLS variant on the activity of the GLS enzyme was assessed in

134 fibroblasts from the patient by quantification of intracellular glutamine and glutamate. The GLS

135 variant indeed resulted in an increased intracellular glutamate:glutamine ratio (Figure. 2A, 2B,

136 S2A). To validate enhanced catalytic activity of the GLS variant, a HEK293 cell model with

137 inducible expression of Ser482Cys-GLS (KGA, the long splice variant) was generated.

138 Induction of Ser482Cys-GLS again strongly increased the glutamate:glutamine ratio while

139 induction of wildtype GLS had no effect (Figure. 2C, 2D, S2B). Inhibition of GLS with CB-

140839 resulted in normalization of glutamate and glutamine concentrations in both fibroblasts and

141 HEK293 cells, providing additional evidence that the Ser482Cys-GLS variant leads to GLS

142 hyperactivity. 
144 Protein expression of both GLS splice variants KGA and GAC in patient fibroblasts was 145 decreased -rather than increased- compared to controls, ruling out that increased GLS activity 146 was due to increased protein availability. Conversely, the observed down-regulation of GLS

147 protein expression suggests it served as a compensatory mechanism aiming at normalizing 148 glutamine and glutamate concentrations (Figure. 2B). In support, introduction of Ser482Cys-

149 GLS in HEK293 cells also evoked decreased GAC expression levels (Figure. 2D, S2C). 150 Furthermore, CB-839-induced GLS inhibition restored GLS expression. GLS expression could 151 also be restored by normalization of glutamate levels through depletion of extracellular 152 glutamine, pointing to glutamate as a regulator of GLS expression (Figure. 2D). Finally, the 153

GLS hyperactivity leads to metabolic compensatory mechanisms observation that protein levels of the reciprocal enzyme GS increased upon expression of Ser482Cys-GLS -an effect that could also be reversed through CB-839-mediated GLS inhibition (Figure. 2B, 2D)- underlines that cellular efforts were aimed at normalizing glutamine and glutamate concentrations.

\section{Ser482 functions as a highly conserved intrinsic restrictor of glutaminase activity}

Ser482 is located near the catalytic site of GLS, but does not have an identified role in the catalytic process itself (12). Alignment of +/- 12.000 GLS protein sequences from $>1000$ genera, revealed that Ser482 is a residue with an extremely high degree of evolutionary conservation (conservation score $>0.98$ ) along with residues directly involved in the catalytic process (Figure. 2E). The Ser482Cys substitution is absent in healthy populations in the databases GoNL(13), gnomAD(14), ClinVar(15) and ExAC(16) and is expected to be tolerated without overall disturbances of the protein fold. Interestingly, substitution by cysteine containing a sterically more demanding and less polar thiol group than serine- changes the electrostatic environment of Tyr466, one of the catalytic residues that protonates glutamine and thereby accelerates deamination into glutamate. This change is likely to enhance the propensity 
168 for proton donation and thereby to increase the speed of the reaction (Figure. 2F, S3,

169 Supplemental discussion).

\section{GLS hyperactivity decreases redox buffer capacity}

171 Oxidative stress is a known consequence of glutamate excess and a common cause of cataract

172 and neuronal injury $(17,18)$. In HEK293 cells expressing Ser482Cys-GLS, clearance of a sub-

173 lethal pulse of hydrogen peroxide was impaired with normal basal reactive oxygen species

174 (ROS) levels. (Figure 3). This indicates that Ser482Cys-GLS results in a lower capacity for 175 ROS scavenging.

176

177

178

180

\section{Ser482Cys-GLS induces lens opacification}

To explore the causal relationship between Ser482Cys-GLS expression and cataract, we examined the effect of this variant in developing zebrafish embryos. Lens transparency at 5 days post fertilization (dpf) in zebrafish embryos injected with Ser482Cys-GLS cDNA was compared to that in control embryos injected with wildtype GLS or uninjected embryos (Figure. S4A). Of the embryos expressing the Ser482Cys-GLS variant, 34/47 (72\%) developed structural opacities in the lens, which were not observed in any of the control embryos (Figure. 4A-C, S4B-D). GLS inhibition with CB-839 from 6 hours post fertilization (hpf) resulted in profoundly decreased formation of structural opacities in the lens of the Ser482Cys-GLS zebrafish embryos (Figure. 4D, Figure. S4E).

\section{Discussion}

We characterize a de novo heterozygous, hyperactive GLS variant found in a patient with infantile onset cataract, skin abnormalities, profound developmental delay and intracerebral glutamate excess. The increased conversion of glutamine into glutamate observed upon introduction of this variant provides a compelling explanation for the strikingly elevated glutamate levels in cerebro and -in view of the central role of glutamate in brain functioning- 
192 likely explains the developmental delay. Furthermore, zebrafish studies unexpectedly reveal

193 that introducing the hypermorphic GLS variant induces lens opacities. Together with the

194 observations that the lens opacities are amenable to GLS inhibition, this supports a role for 195 glutaminase activity in cataract formation.

196 Inborn errors of metabolism are usually due to bi-allelic or mono-allelic loss-of-function 197 variants with few exceptions. Of these, hyperinsulinism-hyperammonemia syndrome is caused 198 by increased sensitivity of the enzyme GDH to allosteric activation and D-2-hydroxyglutaric 199 aciduria is caused by a neomorphic function of the enzyme IDH2(19, 20). The variant described 200 here truly increases enzymatic activity (Supplemental discussion) likely due to an improved 201 electrostatic environment of the GLS catalytic site. To the best of our knowledge, this nature of 202 hypermorphic gain-of-function in which activity is intrinsically increased by improvement of 203 the catalytic machinery has not been described before. Although rare by nature, it is possible 204 that the current paradigm -heterozygous variants in enzyme encoding genes are usually 205 harmless- hampers identification of comparable disease causing hypermorphic variants in 206 enzyme encoding genes.

207 The cellular efforts, aimed at counteracting the effects of the hyperactive enzyme by decreasing 208 GLS protein availability while increasing the reciprocal enzyme GS, underline that increased 209 GLS activity is detrimental. Our data underline the observation by Krebs in 1935 that glutamate 210 acts as a sensor for GLS regulation and reveal that glutamate not only affects GLS enzyme 211 kinetics but also its expression. The extremely high degree of conservation of the hypermorphic 212 residue across $>1000$ genera -comparable only to residues directly involved in the enzymatic 213 conversion of glutamine into glutamate- suggests that the serine residue serves as a built-in 214 restrictor, ensuring submaximal activity rather than maximal enzyme activity of GLS. 
215 A point of interest is the observation that the ratio between glutamate and glutamine was 216 increased in brain and urine, while it remained unaltered in CSF and plasma. We postulate that 217 this discrepancy is explained by the degree to which glutamine and glutamate levels are 218 controlled by GLS. Tissues with abundant expression of GLS -neurons and kidney- are mainly 219 under GLS control (6). The relative importance of GLS within the brain is illustrated by a high 220 glutamate/glutamine ratio (2:1) in normal population (21). GLS overactivity may be masked in 221 other tissues in which GLS is only one of several players -including GS- that together regulate 222 glutamine and glutamate levels. The reduced importance of GLS in plasma is reflected by the 223 significantly lower ratio of glutamate/glutamine (1:15) (22). Further supporting this hypothesis,

224 the ratio is even lower $(\sim 1: 100)$ in CSF, which is produced by choroid plexus from plasma by 225 glial cells that are known to have high GS expression (4) (22). The striking contrast between 226 CSF and brain could be regarded as a cautionary note: CSF should not be readily regarded as a 227 proxy for the brain.

Under physiological conditions, glutamate is important for redox homeostasis as it is the precursor of the anti-oxidant glutathione(2). Glutamate excess, however, is associated with oxidative stress, a common cause of cataract and neuronal damage $(17,18)$. We show that GLS hyperactivity indeed leads to decreased capacity for redox buffering, which can result in oxidative stress. We therefore postulate that glutamate excess contributes to the ophthalmologic phenotype of our patient. In the aqueous humor -nourishing the lens- glutamate concentrations are strictly regulated and even kept low by metabolism and transport(23). Exposure to glutamate causes cataract in chick and rat embryos $(17,24,25)$. In line with the phenotype of the affected patient, zebrafish expressing Ser482Cys-GLS develop lens opacities which could be largely

237 prevented by GLS inhibition. Interestingly, neurons and lens cells are both of ectodermal origin, 238 as is the skin, and share similarities in expression and regulation of glutamate receptors, 239 supporting the notion that disturbed glutamate homeostasis not only affects the brain, but also 
skin and lens(26). Glutamate excitotoxicity has been associated with epilepsy, numerous neurodegenerative diseases, self-injury and agitated behavior(18, 27). The measured glutamate excess in the brain of our patient might therefore provide a plausible explanation for the selfinjury behavior and developmental delay of our patient.

Interestingly, other defects affecting glutamate homeostasis lead to neurological phenotypes as well. Under physiological circumstances, homeostasis of glutamine and glutamate in the brain is strictly regulated by neuronal GLS and astrocytic GS via the glutamine-glutamate shuttle(4).

GLS loss of function variants lead to a phenotypic spectrum. The first description was of a late childhood onset disease, including optic atrophy and spastic ataxia(9). Recently, bi-allelic loss of function variants in GLS were described to cause lethal, neonatal onset encephalopathy characterized by respiratory failure, status epilepticus and early death within weeks after birth(8). These patients had simplified gyral patterns and showed destructions of initially normal appearing brain structures. Both the reported hiccups during pregnancy and the simplified gyral patterns on imaging suggest the damage has its onset prenatally. Given the truncating mutations present in the latter phenotype it is tempting to speculate a dose effect relationship explaining the phenotypic spectrum. This inborn error -together with GLS hyperactivity- illustrates the importance of proper GLS activity for both brain physiology and morphology.

Deficiency of GS -performing the reversed reaction of GLS- results in decreased glutamine levels but normal glutamate levels and hyperammonemia. This has been reported in three individuals which exhibited neonatal encephalopathy, seizures and respiratory failure and early death(28). The absence of epilepsy in our patient with GLS hyperactivity despite increased glutamate levels on brain MRSI is unexpected as glutamate excitotoxicity is considered a critical factor in the initiation of epileptic seizures(29). Seizures can be provoked by either increased glutamate release into the synaptic cleft or decreased re-uptake or recycling from the 
synaptic cleft, which implies that glutamate levels in the synaptic cleft of our patient are

266 unaffected despite overall brain glutamate abundance. The phenotypic neurological spectrum

267 of these patients show the importance of strictly regulated glutamate homeostasis for 268 neurological functioning.

269 A limitation of our study is that only a single patient with a hyperactive variant in GLS could

270 be identified. Hyperactive variants are extremely uncommon, especially in a well conserved 271 catalytic area like in GLS. Intolerance to loss-of-function in GLS is likely high, meaning that 272 mutations will likely be lethal. These factors contribute to a limited patient pool. While definite 273 pathogenic conclusions are considered difficult based on evidence from unique subjects, when

274 adequately studied, these cases can be regarded as experiments of nature and provide invaluable 275 insights. Such is the case here, were we provide strong evidence that GLS hyperactivity causes 276 a new metabolic disorder of glutamate metabolism. Our study furthermore provides insight into 277 the regulation of GLS activity and illustrates the importance of appropriate GLS activity for 278 human brain function, skin and lens transparency.

\section{Materials and Methods}

280 Clinical phenotyping, diagnostics and exome sequencing

281 Clinical phenotyping was performed and diagnostic tests were requested by metabolic 282 pediatricians, clinical geneticists, an ophthalmologist, neurologists and dermatologist. Amino acids analyses in urine were performed on a Biochrom30 analyzer. In the brain, these were determined with quantitative Magnetic Resonance Spectroscopic (MRS) and Spectroscopic Imaging (MRSI) at 1.5Tesla at age 2 and 3 years and with MRSI at 7Tesla at age 14 years.

286 Genetic analysis was performed by trio-based whole-exome sequencing and Sanger 287 sequencing. See supplementary methods for details. 
289 GLS activity was determined in patient fibroblasts and in human embryonic kidney 293 290 (HEK293) cells stably transfected with either wildtype or Ser482Cys-GLS (KGA isoform) or an empty vector, in absence or presence of different concentrations of the allosteric GLS inhibitor CB-839(30). GLS activity was defined as the formation of glutamate from glutamine, quantified by ultra-performance liquid chromatography tandem mass spectrometry(31). Protein 294 expression was assessed by Western Blot.

295

\section{Conservation analysis}

Sequences homologous to human GLS from the non-redundant protein collection at NCBI were aligned in SeaView(32). Obvious partial sequences as well as all pdb sequences were removed which resulted in about 12.000 sequences. The consensus were determined in JalView(33) and fraction of the modal residue in a column were used for generating a color gradients which was mapped onto the GLS structure as a measure of conservation (consensus score).

\section{Reactive oxygen species}

ROS-levels were quantified by flow cytometry (BD FACSCalibur ${ }^{\mathrm{TM}}$ ) as previously described in wildtype GLS or Ser482Cys-GLS transfected HEK293 cells(34).

\section{Animal model}

Zebrafish (Danio rerio) embryos were microinjected at the 1-cell stage with DNA constructs coding for wildtype or Ser482Cys-GLS (KGA isoform). Uninjected zebrafish embryos were used as controls. The embryos were kept under standard laboratory conditions, either in the absence or in the presence of the GLS inhibitor CB-839, prior to assessment of glutamine and glutamate concentrations and lens opacity at 5 days post fertilization (dpf).

\section{Statistics}


311 Statistical analyses were performed by ANOVA, post-hoc Tukey's test using IBM SPSS

312 statistics 21.

314 Study approval

315 The proband's parents provided written informed consent for all aspects of the study.

316 Zebrafish experiments were carried out in accordance with the guidelines of the Animal

317 Experimentation Committee of the Royal Netherlands Academy of Arts and Sciences 318 (KNAW).

319 For more detailed information, see supplementary materials and methods.

320

321

\section{Acknowledgements}

322 We are grateful for the contribution of the patient's family to this study. We would like to thank

323 Willem Hoefakker, Birgit Schiebergen-Bronkhorst, Ans Geboers, Mirjam van Aalderen,

324 Annique Claringbould, Elise Meijer and Lida Lughthart for their great technical assistance. We

325 also thank Sahar Nassirpour and Paul Chang for providing assistance in reconstructing the

326 7Teska MRSI data. This work was funded by ODAS and Erfelijke Stofwisselingsziekten

327 Nederlands taalgebied (ESN).

328 The authors declare no competing financial interests.

\section{Author contributions}

LR, FT, HCMTP, FJTZ, GH, NMVD, JJMJ and PMH contributed to the concept and design of

332 the study. PMH and JJMJ coordinated the study. PJWP, HYK, HCMTP, MSK, GH, NMVD,

333 JJMJ and PMH provided clinical phenotyping, diagnostics, patient care and (genetic)

334 counseling. LR, FT, PJWP, EV, JPW, AAB, SMCS, KJD, MJGB, RJJR, PAWS, DWJK, 
335 GCMB, RLT, JPWB, TBD, HR and FJTZ contributed to the acquisition and interpretation of

336 the data. LR and FT wrote the manuscript and all co-authors critically revised the manuscript 337 and provided final approval of the manuscript to be published.

\section{References}

3391 Curtis, D.R., Phillis, J.W. and Watkins, J.C. (1960) The chemical excitation of spinal neurones 340 by certain acidic amino acids. $J$ Physiol, 150, 656-682.

3412 Lu, S.C. (2009) Regulation of glutathione synthesis. Mol Aspects Med, 30, 42-59.

3423 Nedergaard, M., Takano, T. and Hansen, A.J. (2002) Beyond the role of glutamate as a 343 neurotransmitter. Nat Rev Neurosci, 3, 748-755.

3444 Bak, L.K., Schousboe, A. and Waagepetersen, H.S. (2006) The glutamate/GABA-glutamine 345 cycle: aspects of transport, neurotransmitter homeostasis and ammonia transfer. J Neurochem, 98, 641346653.

3475 Curthoys, N.P. and Watford, M. (1995) Regulation of glutaminase activity and glutamine 348 metabolism. Annu Rev Nutr, 15, 133-159.

3496 Aledo, J.C., Gomez-Fabre, P.M., Olalla, L. and Marquez, J. (2000) Identification of two human 350 glutaminase loci and tissue-specific expression of the two related genes. Mamm Genome, 11, 11073511110.

3527 Spodenkiewicz, M., Diez-Fernandez, C., Rufenacht, V., Gemperle-Britschgi, C. and Haberle, J. 353 (2016) Minireview on Glutamine Synthetase Deficiency, an Ultra-Rare Inborn Error of Amino Acid 354 Biosynthesis. Biology (Basel), 5.

3558 Rumping, L., Büttner, B., Maier, O., Rehmann, H., Lequin, M., Schlump, J., Schmitt, B., 356 Schiebergen-Bronkhorst, B.G.M., Prinsen, B.H. and Losa, M. (UNPUBLISHED (accepted 2018)) Loss 357 of function of GLS disturbs glutamate-glutamine homeostasis and leads to early lethal epileptic 358 encephalopathy. JAMA-NEU, in press.

3599 Lynch, D.S., Chelban, V., Vandrovcova, J., Pittman, A., Wood, N.W. and Houlden, H. (2018) 360 GLS loss of function causes autosomal recessive spastic ataxia and optic atrophy. Ann Clin Transl 361 Neurol, 5, 216-221. 
36210 Kapoor, R.R., Flanagan, S.E., Fulton, P., Chakrapani, A., Chadefaux, B., Ben-Omran, T.,

363 Banerjee, I., Shield, J.P., Ellard, S. and Hussain, K. (2009) Hyperinsulinism-hyperammonaemia 364 syndrome: novel mutations in the GLUD1 gene and genotype-phenotype correlations. Eur J Endocrinol, 365 161, 731-735.

36611 Yang, H., Ye, D., Guan, K.L. and Xiong, Y. (2012) IDH1 and IDH2 mutations in tumorigenesis: 367 mechanistic insights and clinical perspectives. Clin Cancer Res, 18, 5562-5571.

36812 Brown, G., Singer, A., Proudfoot, M., Skarina, T., Kim, Y., Chang, C., Dementieva, I., 369 Kuznetsova, E., Gonzalez, C.F., Joachimiak, A. et al. (2008) Functional and structural characterization 370 of four glutaminases from Escherichia coli and Bacillus subtilis. Biochemistry, 47, 5724-5735.

$371 \quad 13$ Genome of the Netherlands, C. (2014) Whole-genome sequence variation, population structure 372 and demographic history of the Dutch population. Nat Genet, 46, 818-825.

37314 Lek M, K.K., Minikel EV. (Accessed March 20, 2018) 2016:285-291, N. (ed.), in press.

37415 Landrum, M.J., Lee, J.M., Benson, M., Brown, G.R., Chao, C., Chitipiralla, S., Gu, B., Hart, J., 375 Hoffman, D., Jang, W. et al. (2018) ClinVar: improving access to variant interpretations and supporting 376 evidence. Nucleic Acids Res, 46, D1062-D1067.

37716 Lek, M., Karczewski, K.J., Minikel, E.V., Samocha, K.E., Banks, E., Fennell, T., O'Donnell378 Luria, A.H., Ware, J.S., Hill, A.J., Cummings, B.B. et al. (2016) Analysis of protein-coding genetic 379 variation in 60,706 humans. Nature, 536, 285-291.

38017 Spector, A. (1995) Oxidative stress-induced cataract: mechanism of action. FASEB J, 9, 11733811182.

38218 Dong, X.X., Wang, Y. and Qin, Z.H. (2009) Molecular mechanisms of excitotoxicity and their 383 relevance to pathogenesis of neurodegenerative diseases. Acta Pharmacol Sin, 30, 379-387.

38419 Grimaldi, M., Karaca, M., Latini, L., Brioudes, E., Schalch, T. and Maechler, P. (2017) 385 Identification of the molecular dysfunction caused by glutamate dehydrogenase S445L mutation 386 responsible for hyperinsulinism/hyperammonemia. Hum Mol Genet, 26, 3453-3465.

38720 Kranendijk, M., Salomons, G.S., Gibson, K.M., Van Schaftingen, E., Jakobs, C. and Struys, 388 E.A. (2011) A lymphoblast model for IDH2 gain-of-function activity in d-2-hydroxyglutaric aciduria 389 type II: novel avenues for biochemical and therapeutic studies. Biochim Biophys Acta, 1812, 1380-1384. 
391 Kan, H.E. (2015) Multi-center reproducibility of neurochemical profiles in the human brain at 7 Tesla. 392 NMR Biomed, 28, 306-316.

39322 Blau, N., Duran, M. and Gibson, K.M. (2008) Laboratory Guide to the Methods in Biochemical

394 Genetics. Springer, Heidelberg Berlin.

39523 Hu, R.G., Lim, J.C., Kalloniatis, M. and Donaldson, P.J. (2011) Cellular localization of 396 glutamate and glutamine metabolism and transport pathways in the rat ciliary epithelium. Invest 397 Ophthalmol Vis Sci, 52, 3345-3353.

39824 Kawamura, M. and Azuma, N. (1992) Morphological studies on cataract and small lens 399 formation in neonatal rats treated with monosodium-L-glutamate. Ophthalmic Res, 24, 289-297.

40025 Laszczyk, W.A. (1975) Development of Cataract as the Effect of Glutamic Acid Administration 401 to Chick Embryo. Ophthalmic Res, 7, 432-439.

40226 Farooq, M., Kaswala, R.H., Kleiman, N.J., Kasinathan, C. and Frederikse, P.H. (2012) GluA2 403 AMPA glutamate receptor subunit exhibits codon 607 Q/R RNA editing in the lens. Biochem Biophys 404 Res Commun, 418, 273-277.

40527 Brodie, M.J., Besag, F., Ettinger, A.B., Mula, M., Gobbi, G., Comai, S., Aldenkamp, A.P. and 406 Steinhoff, B.J. (2016) Epilepsy, Antiepileptic Drugs, and Aggression: An Evidence-Based Review. 407 Pharmacol Rev, 68, 563-602.

40828 Haberle, J., Gorg, B., Rutsch, F., Schmidt, E., Toutain, A., Benoist, J.F., Gelot, A., Suc, A.L., 409 Hohne, W., Schliess, F. et al. (2005) Congenital glutamine deficiency with glutamine synthetase 410 mutations. $N$ Engl J Med, 353, 1926-1933.

41129 Barker-Haliski, M. and White, H.S. (2015) Glutamatergic Mechanisms Associated with 412 Seizures and Epilepsy. Cold Spring Harb Perspect Med, 5, a022863.

41330 Gross, M.I., Demo, S.D., Dennison, J.B., Chen, L., Chernov-Rogan, T., Goyal, B., Janes, J.R., 414 Laidig, G.J., Lewis, E.R., Li, J. et al. (2014) Antitumor activity of the glutaminase inhibitor CB-839 in 415 triple-negative breast cancer. Mol Cancer Ther, 13, 890-901.

41631 Prinsen, H.C., Schiebergen-Bronkhorst, B.G., Roeleveld, M.W., Jans, J.J., de Sain-van der 417 Velden, M.G., Visser, G., van Hasselt, P.M. and Verhoeven-Duif, N.M. (2016) Rapid quantification of 
underivatized amino acids in plasma by hydrophilic interaction liquid chromatography (HILIC) coupled with tandem mass-spectrometry. J Inherit Metab Dis, 39, 651-660.

42032 Gouy, M., Guindon, S. and Gascuel, O. (2010) SeaView version 4: A multiplatform graphical 421 user interface for sequence alignment and phylogenetic tree building. Mol Biol Evol, 27, 221-224.

42233 Waterhouse, A.M., Procter, J.B., Martin, D.M., Clamp, M. and Barton, G.J. (2009) Jalview 423 Version 2--a multiple sequence alignment editor and analysis workbench. Bioinformatics, 25, 11894241191.

42534 Jelluma, N., Yang, X., Stokoe, D., Evan, G.I., Dansen, T.B. and Haas-Kogan, D.A. (2006) 426 Glucose withdrawal induces oxidative stress followed by apoptosis in glioblastoma cells but not in 427 normal human astrocytes. Mol Cancer Res, 4, 319-330.

42835 Pouwels, P.J., Brockmann, K., Kruse, B., Wilken, B., Wick, M., Hanefeld, F. and Frahm, J. 429 (1999) Regional age dependence of human brain metabolites from infancy to adulthood as detected by 430 quantitative localized proton MRS. Pediatr Res, 46, 474-485.

$43136 \quad$ Kraulis, P.J. (1991) Molscript - a program to produce both detailed and schematic plots of 432 protein structures. Journal of Applied Crystallography, 24, 946-950.

43337 Merritt, E.A. and Murphy, M.E. (1994) Raster3D Version 2.0. A program for photorealistic 434 molecular graphics. Acta Crystallogr D Biol Crystallogr, 50, 869-873.

\section{Figure legends}

Figure 1. Identification of a GLS de novo variant in a patient with bilateral infantile cataract. (A) Photographs of the eyes of the patient at different ages depict a decrease in light reflex, indicating the formation of cataract before the age of three months. (B) Dermatological 439 manifestation of erythematic nodules of approximately $1 \mathrm{~cm}$, here on the dorsum of the foot. 440 (C) Glutamine and glutamate concentrations assessed by Magnetic Resonance Spectroscopy 441 (1.5 Tesla, both STEAM, TR/TM/TE 6000/30/20 ms, and PRESS, TR/TE 3000/30 ms) in the 442 parietal cortex and white matter of the patient at ages 2 and 3 years. The normal range, $+/-2$ 443 SD from mean based on control values of children between 2 and 5 years of age(35) is depicted 
444 in grey. Data represent concentrations in single voxel MRS (o) and in multiple voxels from 445 MRSI (x). (D) Maps of glutamine (middle panel) and glutamate (right panel) levels in the brain 446 of the patient at age 14 years (lower row) and a control (top row), generated from 2D MRSI 447 acquisitions (7T, pulse-acquire, matrix $44 \times 44,0.5 \times 0.5 \times 1.0 \mathrm{~cm}^{3}$, TR/TE $300 / 2.5 \mathrm{~ms}$ ) overlaid 448 on anatomical magnetic resonance images (left panel). (E) Magnetic resonance imaging of the 449 patient at age 16 months, revealing delayed myelination. The transverse T1-weighted image 450 (left) shows the myelinated cerebral white matter as white. The FLAIR (middle) and T2451 weighted (right) images have a lack of contrast between cerebral hemispheric white matter and 452 cortex, indicating that myelination is incomplete. Better myelinated structures, including corpus 453 callosum and internal capsule, have a lower signal. (F) Urinary excretion of glutamate and 454 glutamine, presented as ratios on a logarithmic scale in the urine of the patient (black dots) 455 compared to controls (white dots). (G) DNA Sanger sequencing trio analysis shows the 456 Ser482Cys-GLS de novo variant in the patient, which is absent in the unaffected parents. The 457 underlined sequence indicates the nucleic acid change causing the substitution of the amino 458 acid serine for cysteine. 
A
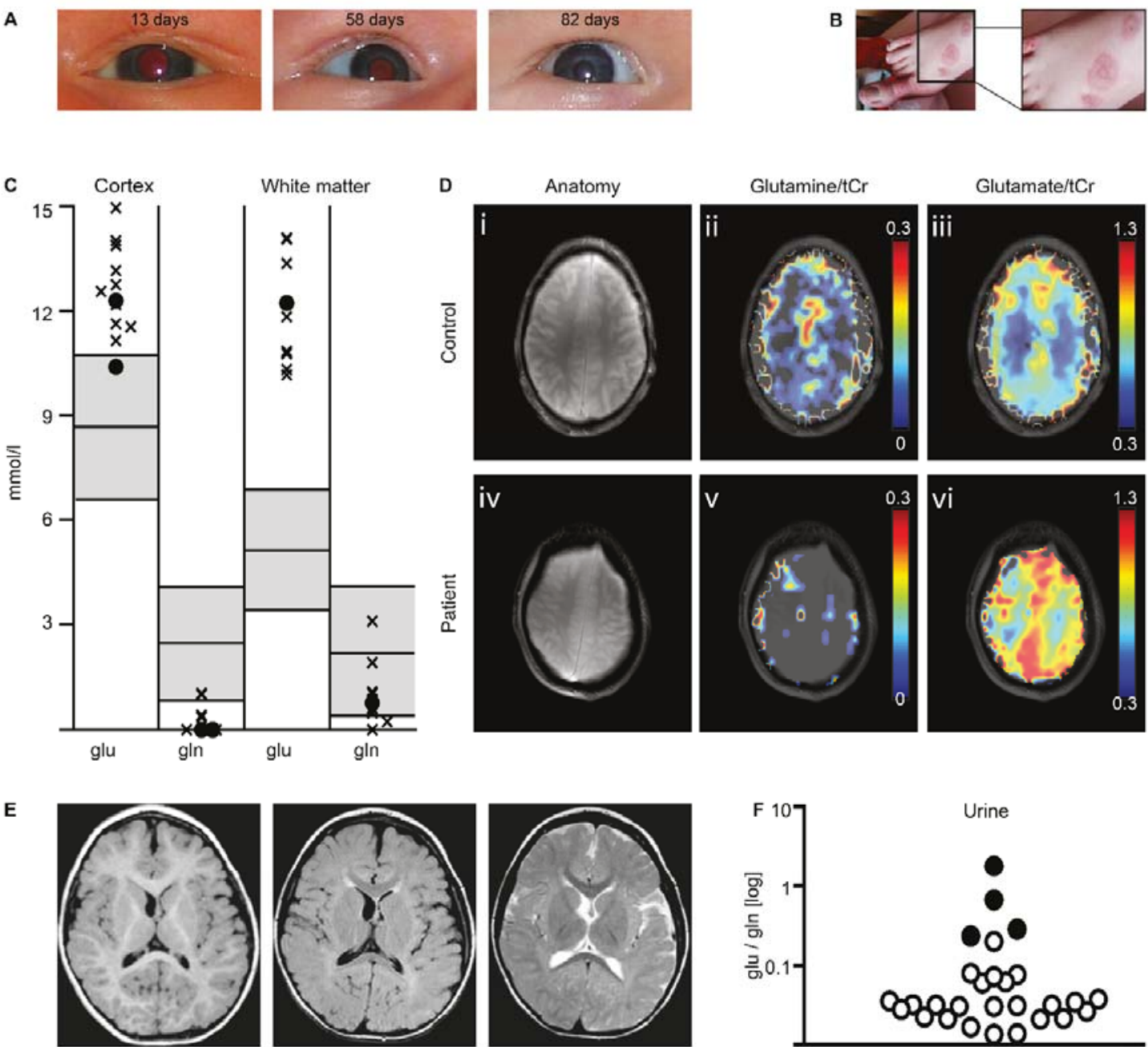

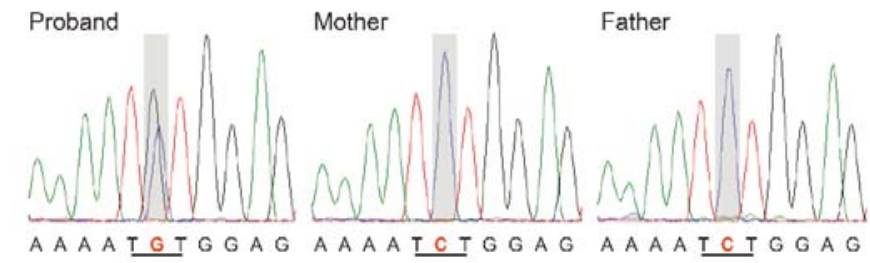


466 Glutamate and glutamine values measured with UPLC-MS/MS, expressed as the ratio of 467 glutamate:glutamine in (A) fibroblasts of 3 controls (white) and the proband expressing 468 Ser482Cys-GLS (black) and (C) HEK293 cells stably transfected with an empty vector 469 (checked), wildtype GLS (white) or Ser482Cys-GLS (black). Cells containing the variant were 470 untreated (highlighted) or treated with $0.1 \mu \mathrm{M}, 0.5 \mu \mathrm{M}, 1 \mu \mathrm{M}$ or $10 \mu \mathrm{M} \mathrm{CB}-839$. Data represent 471 the mean of biological triplicates with standard deviations. ${ }^{*} p<0.05$ (ANOVA, Tukey's test) $472 * * \mathrm{p}<0.01$ (ANOVA, Tukey’s test) ns: not significant. (B-D) Western blots of both GLS splice 473 variants -kidney type glutaminase (KGA) and glutaminase C (GAC)- and GS. (B) In fibroblasts 474 of 3 controls and the patient expressing Ser482Cys-GLS, the latter treated with CB-839 475 corresponding to panel a. The mean of the expression levels in control fibroblasts is arbitrarily 476 set at 1. (D) In HEK293 cells stably transfected with an empty vector (EV), wildtype GLS (Wt) 477 or Ser482Cys-GLS (KGA), the latter treated with CB-839 (corresponding to panel c) or 478 deprived from glutamine to normalize glutamate concentrations ( $g l u=)$. Expression levels in 479 cells expressing wildtype GLS are arbitrarily set at 1. Results are normalized to actin or 480 GADPH. Analyses performed on the same blot are delineated. (E) Conservation analysis of 481 GLS, in which residues with conservation scores from 0 to 0.98 are represented by a color 482 gradient from yellow to red and the most conserved residues $(>0.98)$ are represented in black. 483 These residues are clustered around the catalytic site and most of them are directly involved in 484 the catalytic reaction: Ala339 (0.994), Lys481 (0.992), Asn335 (0.991), Lys289 (0.990), Ser286 485 (0.990), Tyr414 (984), Tyr466 (0.986) and Asn388 (0.983). Among these is Ser482 (0.983), 486 indicated by the asterisk symbol. Glutamine is shown in green ball-an-stick representation. 487 Glycine and proline residues -often conserved for pure structural reasons- were omitted and are 488 shown in light grey. (F) Zoom-in on the catalytic site of GLS in complex with glutamine (green) 489 shows that Ser482 (magenta) is located near the catalytic site. The deamination reaction of 
490 glutamine is initiated by a nucleophilic attack of Ser286 on $\mathrm{C}^{\delta}$ of glutamine (red arrow) and is 491 accelerated by Tyr466 via protonation (black arrows indicate proton transfer). The electrostatic 492 environment of Tyr466 is determined by the hydroxyl-group of Ser482 (yellow dotted line). 493 Hydrogen bonds are shown by dotted black lines. Supplemental Figure. 3 provides additional 494 insight into the enzymatic reaction and the possible consequences of the Ser482Cys 495 substitution. Figures are based on pdb entry $3 \mathrm{vp} 0$ and were generated with molscript(36) and 496 raster3D(37) 


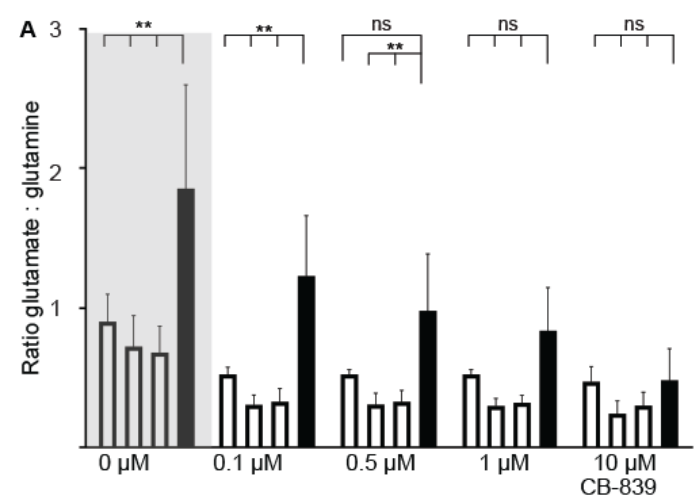

B

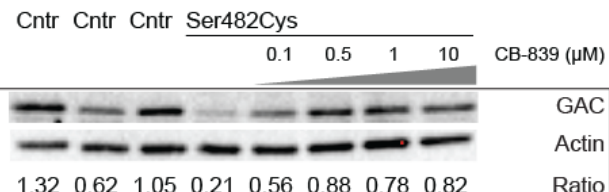

\begin{tabular}{|c|c|c|c|c|c|c|c|c|}
\hline$\infty$ & 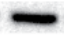 & 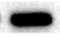 & $=$ & - & - & - & - & KGA \\
\hline 0.81 & 0.94 & 1.25 & 0.43 & 0.46 & 0.61 & 0.63 & 0.66 & Rat \\
\hline 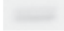 & 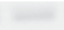 & $=$ & - & - & - & $=$ & $x^{2}$ & \\
\hline 0.97 & 0.92 & 1.11 & 1.76 & 1.63 & 1.58 & 1.50 & 1.15 & \\
\hline
\end{tabular}

E

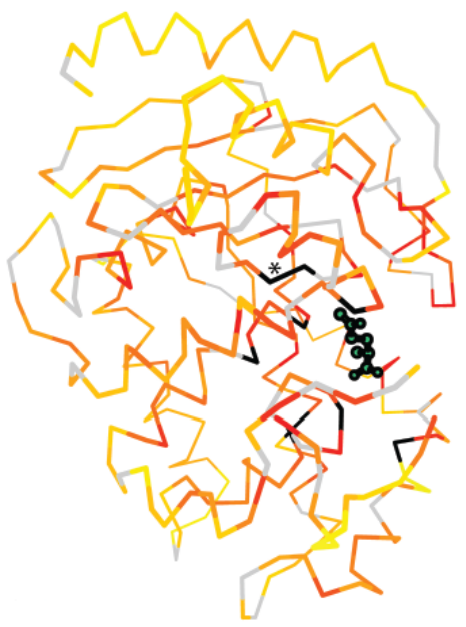

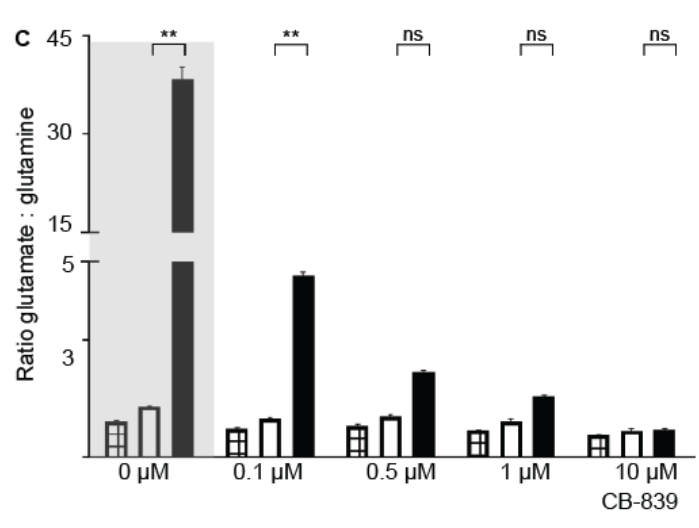

D

EV Wt Ser482Cys

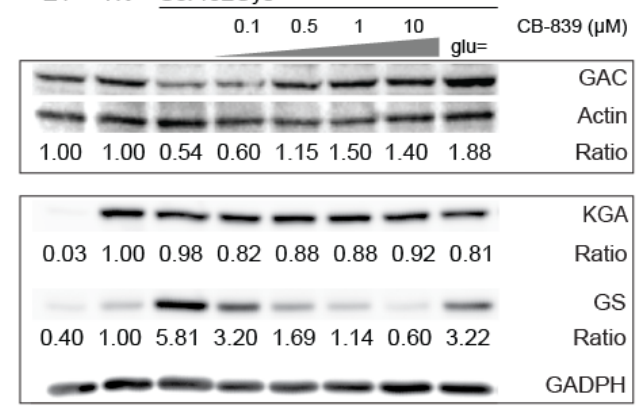

F

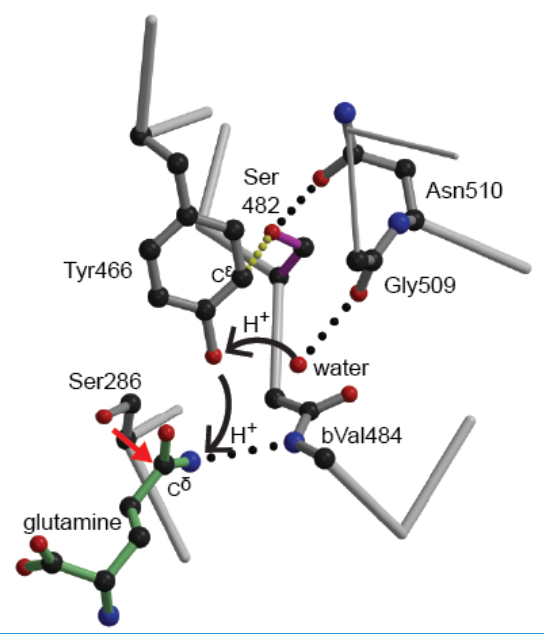


Figure 3. ROS levels and redox buffering capacity. HEK293 cells were transfected with

503 wildtype GLS or Ser482Cys-GLS, with and without exposure to $\mathrm{H}_{2} \mathrm{O}_{2}$, measured with flow

504 cytometry, using CM-H2DCF-DA. (A) Histograms of non-induced (-dox, black) and induced

505 (+dox, grey) cells, showing the shift in $\mathrm{CM}-\mathrm{H}_{2} \mathrm{DCF}$ fluorescence intensity after induction with

506 doxycycline and exposure to $50 \mu \mathrm{M} \mathrm{H}_{2} \mathrm{O}_{2}$, Data are normalized to total cell counts and are

507 representative for biological triplicates. (B) Ratio of the median $\mathrm{CM}-\mathrm{H}_{2} \mathrm{DCF}$ fluorescence

508 intensities of cells induced with doxycycline over uninduced cells. Data present the mean of

509 biological triplicates with standard errors of the mean. ns: not significant $* \mathrm{p}<0.05$ (ANOVA,

510 Tukey's test).

A
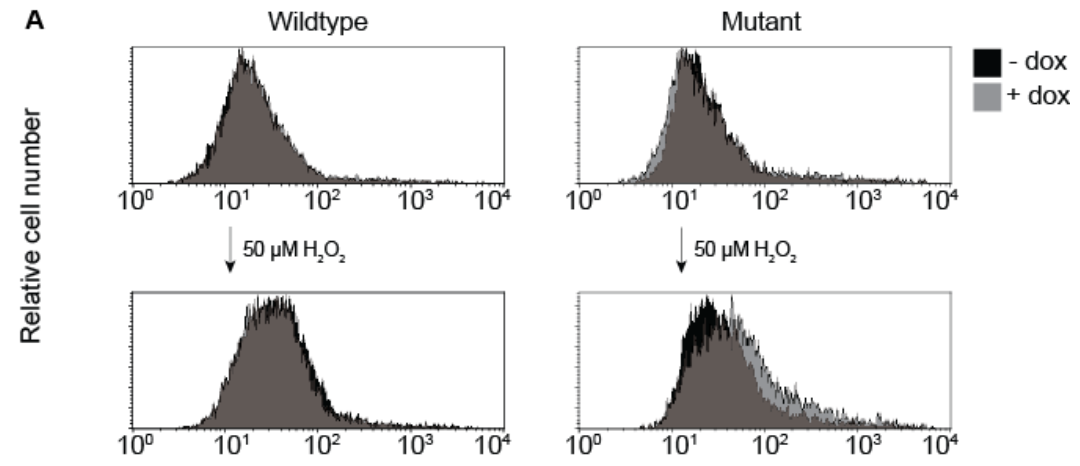

ROS (CM- $\left.\mathrm{H}_{2} \mathrm{DCF}\right)$ fluorescence levels

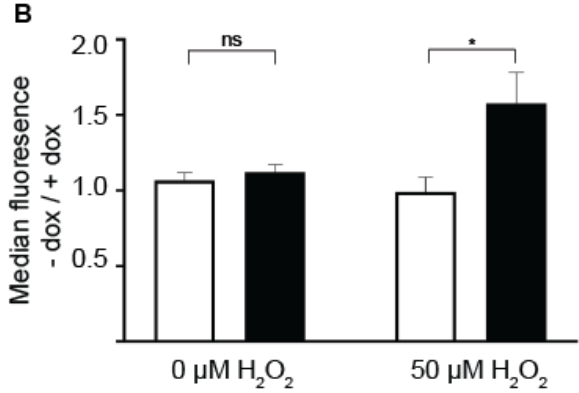


515 Figure 4. Lens opacity of zebrafish expressing Ser482Cys-GLS. Representative images of

516 the lenses of 5 dpf zebrafish embryos of (A) uninjected $n=30$ or injected with vectors containing

517 (B) wildtype GLS cDNA $\mathrm{n}=28$ or (C) Ser482Cys-GLS cDNA KGA isoform n=63.

518 Zebrafish embryos expressing Ser482Cys-GLS were treated with $10 \mu \mathrm{M}$ CB-839 from 6 hpf

$519 n=10$. Opacities in the lens are indicated with arrows. See Supplemental Figure. 4 for all images.

520 Images were obtained with a fluorescence microscope. Linear image editing was performed.
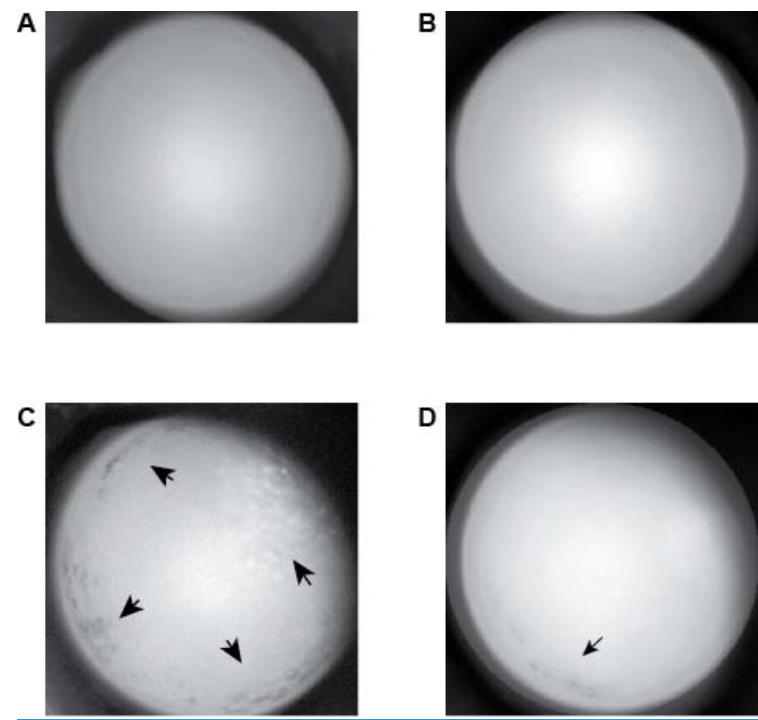

521 